\title{
Emerging transcatheter therapies for tricuspid valve disease
}

\author{
Christine J. Chung, MD, and Isaac George, MD
}

\begin{abstract}
Feature Editor's Introduction-Transcatheter tricuspid valve devices are an emerging group of technologies aimed at reducing the morbidity and mortality associated with severe tricuspid regurgitation (TR). To date, these devices have primarily been implanted in high-risk patients with symptomatic severe functional TR, and feasibility and shortterm results have been reported for a variety of devices.

The devices fall into 2 main categories: repair or replacement devices. The repair devices can be further subdivided into coaptation devices that improve leaflet coaptation, or annuloplasty devices that reshape and downsize the valve annulus. The replacement devices include heterotopic caval valves that reduce caval backflow from severe TR and orthotopic valves that directly re-establish tricuspid valve competence.
\end{abstract}

In this issue of the Journal, Drs Chung and George summarize the current landscape and future directions in transcatheter tricuspid valve interventions. Because isolated tricuspid valve surgery is associated with such poor outcomes, there is considerable interest in less-invasive treatment solutions for symptomatic severe functional TR. Current data from the multicenter TriValve Registry suggest that high procedural success (defined as reduction of TR grade from severe to moderate or less) can be achieved with many of these first-generation devices. Interestingly, many of the more commonly used technologies leverage on repair techniques that have been found to be inferior in surgical series, including edge-to-edge repair (with offlabel use of the MitraClip [(Abbott Vascular, Santa Clara, Calif]) and bicuspidization (with devices such as TriAlign [Mitralign, Tewksbury, Mass] and Tricinch [4Tech Cardio, Galway, Ireland]). Not surprisingly, registry data also suggest that the degree of residual TR after transcatheter tricuspid repair is greater than would be expected with surgical repair.

Clinical application of transcatheter tricuspid valve interventions has largely occurred through uncontrolled feasibility trials and compassionate use. Controlled clinical trials with standardization of patient selection

From Columbia University Medical Center/NewYork-Presbyterian Hospital, New York, NY.

Received for publication March 24, 2020; revisions received March 24, 2020; accepted for publication April 7, 2020; available ahead of print May 26, 2020.

Address for reprints: Isaac George, MD, Columbia University Medical Center/ NewYork-Presbyterian Hospital, 177 Fort Washington Ave, New York, NY 10032 (E-mail: ig2006@cumc.columbia.edu).

JTCVS Open 2020;2:14-9

2666-2736

Copyright $(2020$ The Authors. Published by Elsevier Inc. on behalf of The American Association for Thoracic Surgery. This is an open access article under the CC BY-NCND license (http://creativecommons.org/licenses/by-nc-nd/4.0/).

https://doi.org/10.1016/j.xjon.2020.04.003

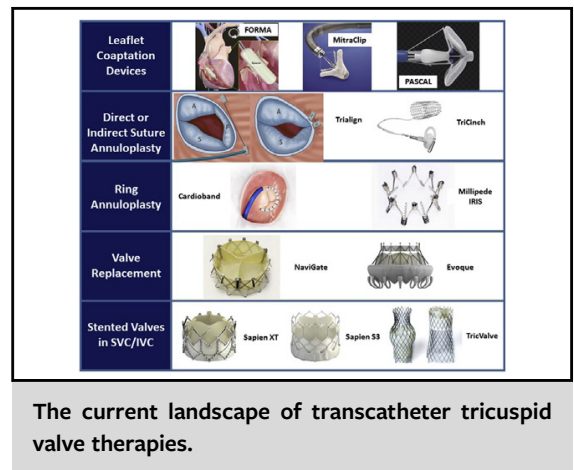

CENTRAL MESSAGE

Functional TR has long been overlooked as a primary target of therapy. Development of percutaneous approaches to treat TR will enable us to more effectively address this unmet clinical need.

See Commentary on page 20.

criteria are needed to define the role of these devices in the future.

\section{Leora B. Balsam, MD}

Tricuspid regurgitation (TR) is most commonly functional, or secondary to left-sided heart disease, atrial fibrillation, and pulmonary hypertension, conditions resulting in right atrial, right ventricular (RV) and tricuspid annular (TA) dilatation and subsequent leaflet tethering. Primary TR, in contrast, is due to congenital or acquired abnormalities of the valve such as rheumatic fever, infective endocarditis, or myxomatous degeneration. Functional TR is often seen as a marker of late-stage chronic heart failure and has long been overlooked as a primary target of therapy. Isolated severe TR is relatively uncommon, and the natural history and clinical influence of TR remain poorly characterized due to its heterogeneous etiologies.

Although it was previously believed that functional TR improves after treatment of left-sided valvular heart disease and thus does not need to be directly addressed, recent data demonstrate that many patients experience ongoing progression of TR after successful treatment of left-sided 
valve disease. ${ }^{1}$ A study of more than 350 patients with isolated TR showed that survival was lower in those with more severe TR (defined by an effective regurgitant orifice area [EROA] of $\geq 40 \mathrm{~mm}^{2}$ ), and lower-than-expected for the general population. Few patients underwent surgery for isolated severe TR. ${ }^{2}$ The high perioperative mortality and adverse outcomes of tricuspid valve surgery likely reflect delayed referral of patients with late-stage heart failure complicated by liver disease and pulmonary hypertension, and highlight current uncertainty and ambivalence over when and how best to treat these patients. Due to ongoing questions regarding criteria for patient selection, and the suboptimal outcomes with surgical intervention, development of less invasive percutaneous treatments for severe TR is highly desirable in addressing this large, unmet clinical need.

\section{TREATMENT OF TR}

Medical management of TR centers on decreasing volume overload and using pulmonary vasodilators to address pulmonary hypertension. Currently, the only Class I indication in both the European and US guidelines for surgical treatment of severe TR is for patients undergoing cardiac surgery for concomitant left-sided lesions such as mitral regurgitation (MR). ${ }^{3}$ However, there is evidence of improved mortality in patients who undergo surgical repair of severe TR before they develop severe New York Heart Association (NYHA) functional class IV symptoms. ${ }^{4}$

The surgical treatment of TR is aimed at restoration of sufficient surface area for leaflet coaptation as well as stabilization of the annulus. Repair of the valve is favored over replacement, although it is associated with an increased risk of persistent and recurrent severe TR. Because severe TR is almost always associated with severe TA dilatation, annuloplasty is the cornerstone of surgical repair techniques. The De Vega technique consists of plication of the TA by placement of 2 semicircular purse string sutures that narrow the anteroposterior dimension of the annulus and decrease the overall effective orifice area. The advantages of this technique over implantation of a ring annuloplasty device include shorter operative time, decreased cost, and avoidance of implantation of foreign material, which may be desirable in cases of infective endocarditis. However, this technique does not restore a normal annular shape and rates of late recurrent TR may be higher. Thus, a variety of incomplete, complete, flexible, and semirigid oval-shaped rings has been designed to preserve the natural configuration of the TA. Successful implantation of a prosthetic ring requires avoiding injury to the adjacent aortic valve, right coronary artery, and the bundle of His.

The benefit of replacing rather than repairing the tricuspid valve is less residual TR, but the loss of a lowpressure outlet can result in acute postoperative RV failure.
It remains unclear which baseline patient characteristics best predict this adverse outcome. Furthermore, the risk of thrombosis in the tricuspid position is high. Despite these concerns, patients with a very large TA and significant tethering of the leaflets are likely better suited to valve replacement rather than repair. Careful sizing of the prosthetic valve is necessary to minimize distortion and disturbance of flow in the right coronary artery.

\section{REVIEW OF EMERGING TRANSCATHETER THERAPIES FOR TR}

The aim of this review is to provide a broad overview of the percutaneous therapies currently in development for the treatment of TR, focusing on devices that have already been used in human studies. The anatomic targets of most transcatheter tricuspid valve repair devices are either the leaflets or the annulus. Thus, current devices can be broadly grouped into coaptation or annuloplasty devices. A third category of caval implants is designed to mitigate the adverse hemodynamic effects of TR on the liver and decrease systemic venous congestion. Finally, several transcatheter tricuspid valve replacement (TTVR) devices have progressed beyond first-in-man experiences to larger early feasibility studies. See Figure 1 for a visual overview of tricuspid valve therapies.

\section{COAPTATION DEVICES}

The off-label use of the MitraClip System (Abbott Vascular, Santa Clara, Calif) has become the first choice for many high-risk patients with severe functional TR. However, the edge-to-edge repair technique is hindered in the tricuspid position by more challenging transesophageal echocardiographic imaging of the valve. Furthermore, the coaptation gaps in patients with functional TR are often much larger than those in patients with MR, which necessitates the use of multiple clips. The best results are often seen with approximation of the anterior and/or posterior leaflet with the septal leaflet. Creation of a valve with a triple orifice by using multiple clips to connect the septal with both the anterior and posterior leaflets has also been described. Approximation of the anterior and posterior leaflets is generally avoided because it may distort the valve and worsen TR. A study of 42 high-risk patients with isolated TR or combined TR and MR reported successful leaflet approximation in $83 \%$ ( 35 of 42 patients) utilizing a total of 68 clips, with the vast majority placed in the anteroseptal commissure. In those with procedural success, there was a significant $62.5 \%$ reduction in EROA (from $0.8 \mathrm{~cm}^{2}$ to $0.3 \mathrm{~cm}^{2}$ ) and improvement in 6-minute walking distance. ${ }^{5}$ Currently, the Trial to Evaluate Treatment With Abbott Transcatheter Clip Repair System in Patients With Moderate or Greater Tricuspid Regurgitation (TRILUMINATE) study (NCT03227757) is enrolling patients with symptomatic moderate or greater TR at 25 sites 


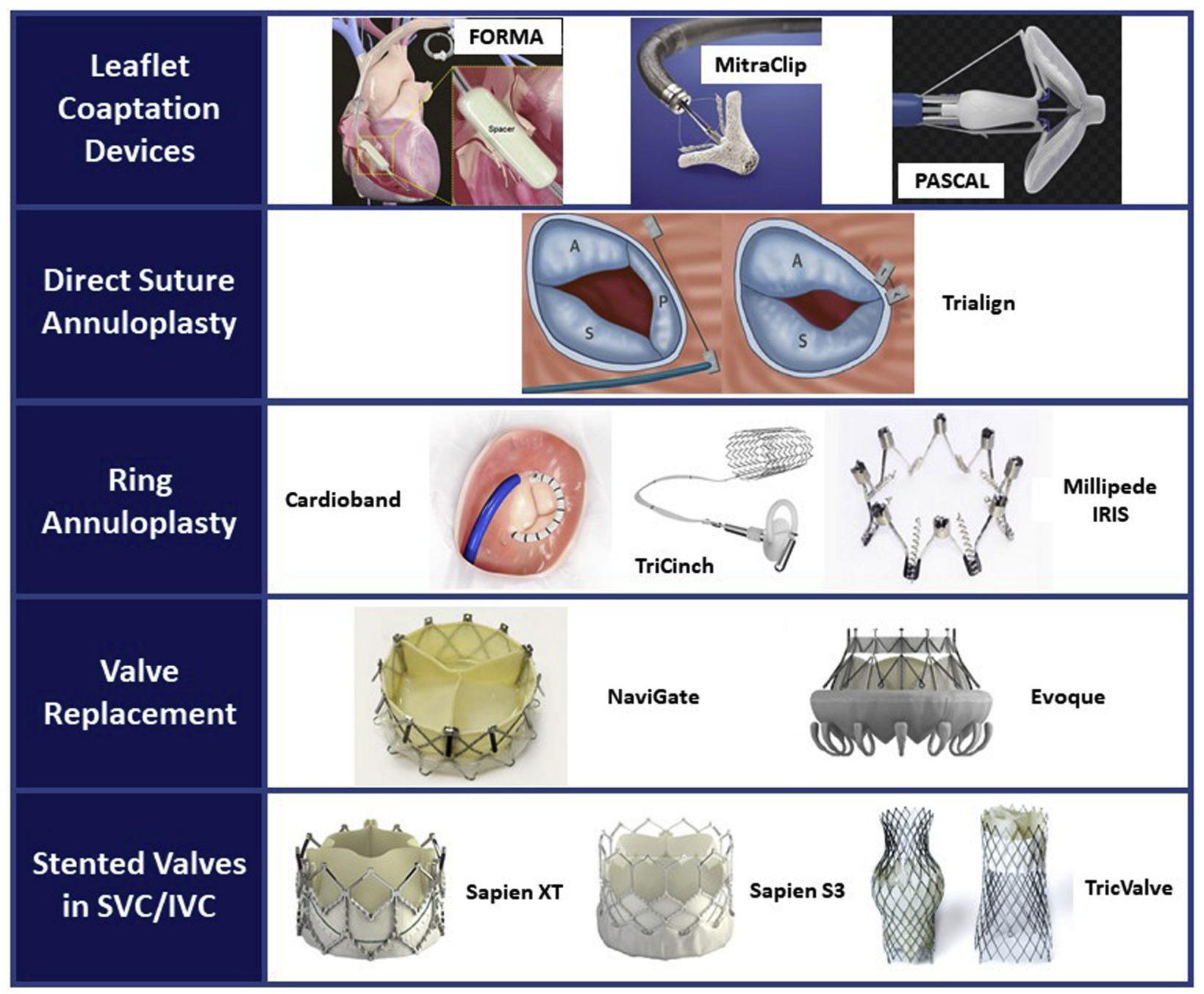

FIGURE 1. The current landscape of transcatheter tricuspid valve therapies. $S V C$, Superior vena cava; $I V C$, inferior vena cava.

in Europe, Canada, and the United States with up to 3 years of follow-up to evaluate this approach.

Another leaflet coaptation device aimed at edge-to-edge repair is the Pascal System (Edwards Lifesciences, Irvine, Calif), which is composed of 2 paddles approximately $25 \mathrm{~mm}$ in width that are capable of independently grasping the leaflets, as well as a $10-\mathrm{mm}$ central spacer intended to fill and thereby reduce the EROA. The first use of the Pascal device for treatment of TR was reported during early 2018. Placement of the first device on the anterior and septal leaflets was followed by placement of a second device on the posterior and septal leaflets, resulting in reduction of TR from torrential to moderate with a mean transtricuspid gradient of $1 \mathrm{~mm} \mathrm{Hg}$. At $1 \mathrm{month}$, the patient had improvement in signs of systemic venous congestion, NYHA functional class from IV to II, greater 6-minute walking distance, and better quality of life scores. ${ }^{6}$ The Edwards PASCAL Transcatheter Valve Repair System in Tricuspid
Regurgitation (CLASP TR) Early Feasibility Study (NCT03745313) is currently nearing enrollment of a cohort of 45 patients to demonstrate the early safety and efficacy of this device.

The Forma Repair System (Edwards Lifesciences) is designed to increase the surface area for leaflet coaptation by placing a foam-filled balloon spacer within the regurgitant orifice. The device is advanced via the left subclavian or axillary vein through a $20 \mathrm{~F}$ to $24 \mathrm{~F}$ introducer sheath and placed within the annulus over a rail that is anchored to the septal aspect of the RV apex. Since the initial first-inman use of the device in 2015, 15 of 18 patients treated under compassionate use have reported 1-year clinical follow-up. Successful implantation was achieved in 16 of 18 patients, with 2 patients experiencing RV perforation requiring open surgical rescue. There were significant improvements in NYHA functional status, 6-minute walking distance, and quality of life scores. At 1 year, there was 
reduction in TR from severe in $94 \%$ of patients to moderate to severe or less in $46 \%$ of the cohort with residual moderate TR in most cases. ${ }^{7}$ Next-generation Forma devices are being developed to minimize the risk of dislodgement and perforation, which occurred in 2 patients in this initial cohort. ${ }^{8}$ However, the Repair of Tricuspid Valve Regurgitation Using the Edwards Tricuspid Transcatheter Repair System (SPACER) trial (NCT02787408) designed to assess the safety and efficacy of the Forma device in patients with significant symptomatic TR at high surgical risk has now been closed.

\section{ANNULOPLASTY DEVICES}

Transcatheter annular-based systems can be categorized as direct suture or ring annuloplasty devices. The TriAlign device (Mitralign, Tewksbury, Mass) is a transcatheter annuloplasty system that mimics the surgical Kay technique, in which multiple sutures placed across the posterior leaflet effectively obliterate it, resulting in bicuspidization of the valve. A transjugular approach is used to advance an insulated radiofrequency wire across the annulus. Two pledgets are fixed at the posteroseptal and anteroposterior commissures and subsequently cinched together using a plication lock device, resulting in posterior leaflet plication and tricuspid valve bicuspidization. The first multicenter experience of 15 patients with moderate or greater TR demonstrated $100 \%$ success with initial device implantation but with 3 occurrences of detachment of a single pledget. There were significant reductions in annular dimensions $\left(12.3 \mathrm{~cm}^{2}\right.$ to $\left.11.3 \mathrm{~cm}^{2}\right)$ and EROA $\left(0.51 \mathrm{~cm}^{2}\right.$ to $\left.0.32 \mathrm{~cm}^{2}\right)$, as well as significant improvement in NYHA functional class $(\geq 1$ class) and 6-minute walk test (245 to $298 \mathrm{~m}$ ). ${ }^{9}$ The ongoing Safety and Performance of the Trialign Percutaneous Tricuspid Valve Annuloplasty System (PTVAS) for Symptomatic Chronic Functional Tricuspid Regurgitation (SCOUT II) study (NCT03225612) will follow 60 patients in the United States and Europe for up to 5 years.

The TriCinch Coil System (4Tech Cardio, Galway, Ireland) is the second-generation iteration of a device that also replicates the Kay annuloplasty procedure by decreasing the anteroposterior commissure. An epicardial coil is delivered in the pericardial space in the midanterior portion of the tricuspid annulus and used to tether a selfexpanding stent deployed in the subhepatic region of the inferior vena cava. Preliminary data from the first $24 \mathrm{pa}-$ tients treated with the first-generation device revealed successful implantation in $81 \%$ of patients with significant ( $\geq 1$ grade) reduction in acute TR severity in $94 \%$. However, 5 patients $(23 \%)$ experienced late detachment of the anchor, prompting the changes in design of the currentgeneration device. $^{10}$

The Cardioband system (Edwards Lifesciences) is an adjustable Dacron (Dupont, Wilmington, Del) band using up to 17 anchors deployed on the atrial side of the TA to fix the device. Cinching of the band provides a controlled reduction of the anteroposterior and septolateral TA dimensions. The safety and efficacy of this device in the treatment of 30 patients with moderate to severe functional TR who were deemed inoperable was recently reported. There was $100 \%$ technical success. At 6 months, there were significant average reductions in the septolateral diameters of $9 \%$ (42 $\mathrm{mm}$ vs $38 \mathrm{~mm}$ ), in the proximal isovelocity surface area EROA of $50 \%\left(0.8 \mathrm{~cm}^{2}\right.$ vs $0.4 \mathrm{~cm}^{2}$ ), and in mean vena contracta width of $28 \%$ $(1.2 \mathrm{~cm}$ vs $0.9 \mathrm{~cm})$. The majority of patients experienced improvement in NYHA functional class, with $88 \%$ reporting NYHA class I or II symptoms at 6 months, compared with $82 \%$ with NYHA class III to IV symptoms at baseline, as well as in 6-minute walk distance and quality of life surveys. ${ }^{11}$

The Millipede IRIS system (Boston Scientific, Marlborough, Mass) is a complete, adjustable, semirigid annuloplasty ring that was designed for the treatment of functional MR and is now being tested for treatment of TR. The device is composed of a zigzag-shaped nitinol ring with corkscrew anchors that attach it to the fibrous annulus. Contraction of the device results in closer approximation of the screws and subsequent reduction in the diameter of the circular frame. The Millipede has been surgically implanted in the TV in 2 patients undergoing concomitant treatment of MR, and showed a reduction in TA diameter of nearly $40 \%$ with only trace TR that was sustained at 1 year. A dedicated transcatheter delivery system for the tricuspid valve is currently being developed.

\section{HETEROTOPIC CAVAL IMPLANTS}

Heterotopic caval valve implants aim to mitigate the systemic venous congestion caused by backflow from severe TR rather than addressing the incompetence of the valve directly. Two different types of devices have been used for caval implantation: balloon-expandable devices originally intended for use in transcatheter aortic valve replacement and dedicated self-expandable devices designed specifically for caval implantation.

The feasibility, safety and efficacy of this approach was described in 25 patients with severe symptomatic TR deemed unsuitable for surgery. The majority of patients were treated with a single valve in the inferior vena cava and $72 \%$ received off-label use of the Sapien XT and Sapien 3 balloon-expandable valves (Edwards Lifesciences). Due to the capacity of the inferior vena cava to dilate up to $35 \mathrm{~mm}$, preparation of the landing zone with prestenting or surgical banding are required when using these valves in the vena cava. Six patients received the TricValve (P\&F Products and Features GmbH, Wessling, Germany), a dedicated self-expanding pericardial valve mounted on a nitinol stent with little radial force specifically designed for the low-pressure system on the right side of the heart, 


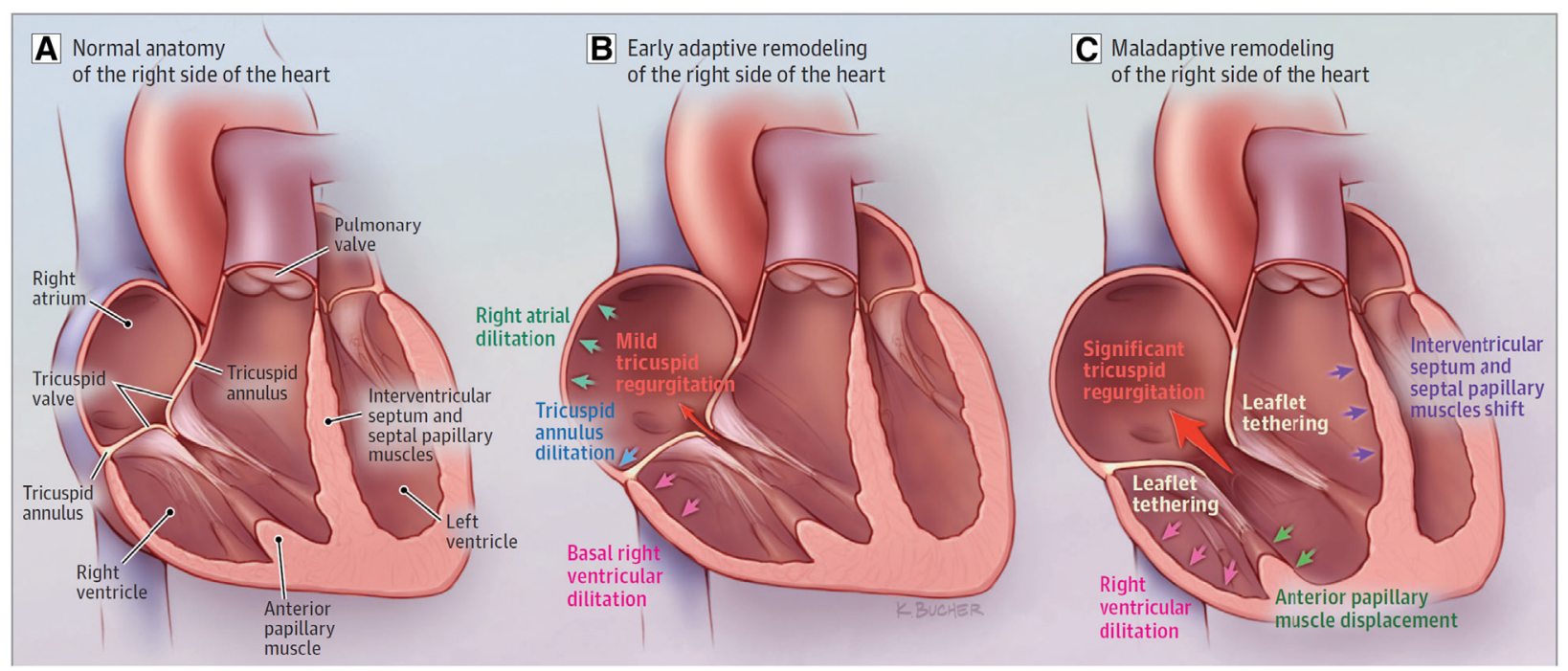

FIGURE 2. Anatomic changes associated with the progression of tricuspid regurgitation. A, Normal anatomy of the right side of the heart. B, Early adaptive remodeling of the right side of the heart. C, Maladaptive remodeling of the right side of the heart. Source: Hahn RT, Waxman AB, Denti P, Delhaas T. Anatomic relationship of the complex tricuspid valve, right ventricle, and pulmonary vasculature: a review. JAMA Cardiol. 2019;4:478-87.

obviating the need for prestenting. This multicenter registry reported $96 \%$ procedural success with 2 instances of device migration requiring surgical retrieval. Thirty-day and inhospital mortality were $8 \%(2$ of 25$)$ and $16 \%$ (4 of 25$){ }^{12}$

\section{TTVR}

Devices specifically designed for TTVR are still in early stages of development. There are multiple challenges affecting early-generation devices: the need for very large bore access, arising from the significantly larger annular area of the tricuspid compared with the aortic and mitral valves; valve anchoring in a large, dynamic annulus that lacks calcification; the need for multimodality imaging of the tricuspid valve; and interaction with pacing leads and the native conduction system because the bundle of His lies near the commissure between the septal and anterior tricuspid valve leaflets. Furthermore, because the right-sided circulation is such a low-pressure system, the risk of valve thrombosis in the tricuspid position is high, and patients will likely require long-term, if not indefinite, anticoagulation therapy. Additionally, abrupt abrogation of significant TR can acutely increase RV afterload, and careful selection of patients to minimize the risk of RV decompensation will be important to ensuring favorable outcomes.

The NaviGate valve (NaviGate Cardiac Structures, Inc, Lake Forest, Calif) is a self-expanding valve composed of a nitinol alloy stent with a mounted trileaflet equine pericardial valve. The inferior, or ventricular, aspect of the valve has a larger diameter than the superior, or atrial, aspect of the valve, and is designed to engage the annulus and leaflets from both aspects while minimizing extension into the chambers and mimicking the geometry of a dilated annulus typically found in functional TR. Initial feasibility studies have shown that the valve has good hemodynamic performance. In 27 cases of first-in-human implantations of the device using both transjugular and transatrial approaches, there was significant reduction in TR from severe/torrential to $\leq 2$ in all patients with $78 \%$ having none or trivial residual TR. ${ }^{13}$

\section{CONCLUSIONS}

TR is a prevalent condition whose long-term sequelae have long been underappreciated and undertreated. The nascent field of transcatheter interventions to treat TR has demonstrated promising results. The TriValve Registry of more than 300 high-risk patients with severe TR who were treated at 18 centers with MitraClip, Pascal, Cardioband, first-generation TriCinch, Trialign, Forma, and NaviGate reported encouraging procedural success rates $(72.8 \%)$, low 30 -day mortality (3.6\%), and significant clinical improvement. ${ }^{14}$ Multiple additional devices, such as the Evoque valve (Edwards Lifesciences), are planned for first-in-man studies in the near future.

The significant capacity of the TA to dilate, with resultant tethering of leaflets and large coaptation gaps, makes application of both leaflet and annuloplasty devices more technically challenging and raises concern for late recurrence of TR (Figure 2). When patients present with early stages of TR with preserved or only mild TA and primarily basal RV dilatation, use of either a leaflet or an annuloplasty device is likely to be successful in reducing the severity of TR. However, once patients have developed later stages of severe TR with significant RV dilatation and leaflet tethering, a leaflet device may not be feasible and there would be 
concern for leaflet detachment as well as later recurrence of severe TR. Once there is massive or torrential TR with severe leaflet tethering, often the only options are a replacement valve or consideration of a palliative caval valve. Further studies of these devices will enable refinement of patient selection criteria and shed light on the anatomic features predictive of both immediate procedural success and long-term durability.

\section{Conflict of Interest Statement}

The authors reported no conflicts of interest.

The Journal policy requires editors and reviewers to disclose conflicts of interest and to decline handling or reviewing manuscripts for which they may have a conflict of interest. The editors and reviewers of this article have no conflicts of interest.

\section{References}

1. Parolari A, Barili F, Pilozzi A, Pacini D. Ring or suture annuloplasty for tricuspid regurgitation? A meta-analysis review. Ann Thorac Surg. 2014;98: 2255-63.

2. Topilsky Y, Nkomo VT, Vatury O, Michelena HI, Letourneau T, Suri RM, et al. Clinical outcome of isolated tricuspid regurgitation. JACC Cardiovasc Imaging. 2014;7:1185-94.

3. Nishimura RA, Otto CM, Bonow RO, Carabello BA, Erwin JP, Fleisher LA, et al. 2017 AHA/ACC focused update of the 2014 AHA/ACC guideline for the management of patients with valvular heart disease: a report of the American College of Cardiology/American Heart Association task force on clinical practice guidelines. J Am Coll Cardiol. 2017;70:252-89.
4. Topilsky Y, Khanna AD, Oh JK, Nishimura RA, Enriquez-Sarano M, Jeon YB, et al. Preoperative factors associated with adverse outcome after tricuspid valve replacement. Circulation. 2011;123:1929-39.

5. Lurz P, Besler C, Noack T, Forner AF, Bevilacqua C, Seeburger J, et al. Transcatheter treatment of tricuspid regurgitation using edge-to-edge repair: procedural results, clinical implications and predictors of success. EuroIntervention. 2018;14:e290-7.

6. Fam NP, Ho EC, Zahrani M, Samargandy S, Connelly KA. Transcatheter tricuspid valve repair with the Pascal System. JACC Cardiovasc Interv. 2018; 11:407-8.

7. Perlman G, Praz F, Puri R, Ofek H, Ye J, Philippon F, et al. Transcatheter tricuspid valve repair with a new transcatheter coaptation system for the treatment of severe tricuspid regurgitation: 1-year clinical and echocardiographic results. JACC Cardiovasc Interv. 2017;10:1994-2003.

8. Asmarats L, Philippon F, Bédard E, Rodés-Cabau J. Forma tricuspid repair system: device enhancements and initial experience. EuroIntervention. 2019;14:1656-7.

9. Hahn RT, Meduri CU, Davidson CJ, Lim S, Nazif TM, Ricciardi MJ, et al. Early feasibility study of a transcatheter tricuspid valve annuloplasty: SCOUT trial 30day results. J Am Coll Cardiol. 2017;69:1795-806.

10. Gheorghe L, Swaans M, Denti P, Rensing B, Van der Heyden J. Transcathete tricuspid valve repair with a novel cinching system. JACC Cardiovasc Interv. 2018;11:e199-201.

11. Nickenig G, Weber M, Schueler R, Hausleiter J, Näbauer M, von Bardeleben RS et al. 6-Month outcomes of tricuspid valve reconstruction for patients with severe tricuspid regurgitation. J Am Coll Cardiol. 2019;73:1905-15.

12. Lauten A, Figulla HR, Unbehaun A, Fam N, Schofer J, Doenst T, et al. Interventional treatment of severe tricuspid regurgitation: early clinical experience in a multicenter, observational, first-in-man study. Circ Cardiovasc Interv. 2018;11: e006061.

13. Navia JL, Kapadia S, Elgharably H, Harb SC, Krishnaswamy A, Unai S, et al First-in-human implantations of the NaviGate Bioprosthesis in a severely dilated tricuspid annulus and in a failed tricuspid annuloplasty ring. Circ Cardiovasc Interv. 2017;10:e005840.

14. Taramasso M, Alessandrini H, Latib A, Asami M, Attinger-Toller A, Biasco L, et al. Outcomes after current transcatheter tricuspid valve intervention: midterm results from the international TriValve registry. JACC Cardiovasc Interv 2019;12:155-65. 